\title{
ANDES

\section{Estudio genético de acuerdo a características fenotípicas de niños y adolescentes con discapacidad intelectual de etiología indeterminada}

\author{
Children and adolescents with intellectual disabilities studied with genetic \\ tests according to their clinical phenotype
}

\author{
Paola Santander ${ }^{\mathrm{a}, \mathrm{b}, \mathrm{c}}$, María José Pedemonte ${ }^{\mathrm{d}}$, Mónica Troncoso ${ }^{\mathrm{a}, \mathrm{b}}$, Carolina Yáñez ${ }^{\mathrm{a}, \mathrm{b}}$, \\ María Alejandra Cárdenas ${ }^{\mathrm{d}}$, Karen Guajardo ${ }^{\mathrm{b}}$, Carolina Silva ${ }^{\mathrm{b}}, \mathrm{Macarena}$ Neves $^{\mathrm{b}}$, \\ Claudia López $^{\mathrm{a}, \mathrm{b}}$, Paola Lagos ${ }^{\mathrm{b}}$, Andrés Barrios ${ }^{\mathrm{a}, \mathrm{b}}$, Ledia Troncoso ${ }^{\mathrm{a}, \mathrm{b}}$
}

\author{
aDepartamento de Pediatría y Cirugía Infantil Centro, Facultad de Medicina, Universidad de Chile. Santiago, Chile. \\ bervicio de Neuropsiquiatría Infantil, Hospital Clínico San Borja Arriarán. Santiago, Chile. \\ 'Grupo de Trabajo en Discapacidad Intelectual y Salud, Facultad de Medicina, Universidad de Chile. Santiago, Chile. \\ dPrograma de Título de Especialistas en Neurología Pediátrica, Facultad de Medicina, Campus Centro, Universidad de Chile. Santiago, Chile.
}

Recibido: 10 de julio de 2020; Aceptado: 07 de junio de 2021

\section{¿Qué se sabe del tema que trata este estudio?}

La discapacidad intelectual afecta al 1-3\% de la población mundial. Es un trastorno del neurodesarrollo que compromete el funcionamiento intelectual y su capacidad adaptativa, que abarca habilidades conceptuales, sociales y prácticas. Las etiologías son múltiples, y su abordaje puede ser complejo. Existe un grupo de pacientes con etiología indeterminada que es de probable origen genético.

\section{¿Qué aporta este estudio a lo ya conocido?}

Este estudio presenta aspectos clínicos y evaluaciones de funcionamiento cognitivo de un grupo de niños y adolescentes con diagnóstico de discapacidad intelectual de causa indeterminada, abordando el estudio con paneles genéticos y microarray cromosómico en relación a las características fenotípicas de los pacientes.

\section{Resumen}

La discapacidad intelectual (DI) es un trastorno del neurodesarrollo manifestado por limitaciones en el funcionamiento intelectual y adaptativo, de etiologías diversas, dentro de las cuales se incluyen las causas genéticas. Objetivo: Describir estudios genéticos realizados en una serie de niños y adolescentes con DI de etiología previamente indeterminada, considerando sus características fenotípicas. Pacientes y Método: Estudio descriptivo de una serie de pacientes con DI de 6 a 18 años. Se revisaron registros clínicos, resultados de evaluación cognitiva (Wechsler-TADI) y estudio genético realizado. Se clasificaron según características fenotípicas en: Grupo 1 pacientes sin fenotipo específico, Grupo 2: pacientes con fenotipo de desórdenes del neurodesarrollo Angelman-Rett like y Grupo 3: pacientes con epilepsia de difícil manejo. Grupo 1 se estudió con microarray cromosómico y Grupos 2 y
Palabras clave:

Discapacidad

Intelectual;

Neurodesarrollo;

Desarrollo Psicomotor;

Panel Genético

Correspondencia:

Paola Santander

paosantander@gmail.com 
3 con paneles genéticos específicos. Resultados: Se describen 18 pacientes, edad promedio 11 años, predominio sexo masculino, de padres no consanguíneos, con antecedente de retraso psicomotor. Comorbilidades frecuentes: epilepsia, rasgos de trastorno espectro autista (TEA) y dificultades conductuales. La mayoría tuvo examen neurológico sin focalidad y TADI con edades de desarrollo muy descendidas. En Grupo 1 se diagnosticó un paciente con una microdeleción 16p11.2 y en Grupo 3 se diagnosticó una duplicación del gen IQSEC2, en un paciente epiléptico de difícil manejo. Conclusiones: Las características fenotípicas permiten orientar la elección de estudios genéticos específicos en niños y adolescentes con DI de etiología previamente indeterminada para acercarse al diagnóstico etiológico.
Keywords:

Intellectual Disability;

Neurodevelopment;

Psychomotor

Development;

Genetic Testing Panels

\section{Introducción}

La discapacidad intelectual (DI) es un trastorno del desarrollo que determina compromiso del comportamiento adaptativo en los dominios conceptual, social y práctico, junto a limitaciones del funcionamiento intelectual que resulta ser significativamente inferior al promedio confirmado mediante evaluación clínica y test estandarizados ${ }^{1-5}$.

Desde la infancia temprana, las personas con DI presentan retraso del desarrollo psicomotor (RDSM) aislado o asociado a otras condiciones neurológicas, como epilepsia y trastorno del espectro autista (TEA). A pesar de los avances en encontrar la causa, en un grupo importante de pacientes con DI no se encuentra etiología identificable. La etiología de DI es variada y las causas genéticas se estiman en $50 \%$ de los pacientes, identificándose más de 700 genes asociados con diferentes tipos de herencia ${ }^{6-9}$.

El desarrollo de nuevas técnicas de estudios genéticos ha aumentado el rendimiento diagnóstico en pacientes con $\mathrm{DI}^{10,11}$. La nueva generación de estudios incluye el desarrollo de paneles genéticos, que han sido de utilidad en dirigir el estudio a patologías específicas, y el microarray cromosómico (CMA, por su sigla en inglés), que pesquisa en casos de DI un 15 a $30 \%$ variaciones en el número de copias (microdeleciones, microduplicaciones $)^{12,13}$. Esto sumado a técnicas de secuenciación exómica y genómica completa han aportado en conjunto a nuevas detecciones de causas genéticas y han ampliado el espectro fenotípico de cuadros genéticos con etiología conocida ${ }^{14-16}$.

El objetivo de este estudio es describir los resultados de estudios genéticos realizados en una serie de niños y adolescentes con discapacidad intelectual de etiología previamente indeterminada, considerando sus características fenotípicas.

\section{Pacientes y Método}

Se diseñó un estudio descriptivo. Se incluyeron niños y adolescentes con diagnóstico de DI a quienes se les realizó estudio etiológico previo donde no se encuentra causa identificable, clasificándose como casos de DI de etiología indeterminada. El estudio se realizó 
en pacientes entre 6 a 18 años de edad evaluados entre enero 2018 a enero 2020, por el equipo de Neurólogos Infantiles en el policlínico del Servicio de Neuropsiquiatría Infantil del Hospital Clínico San Borja Arriarán (HCSBA), Santiago, Chile. Se realizaron evaluaciones clínicas presenciales y revisión de registros clínicos, registrando sexo, edad, antecedentes familiares de DI y consanguinidad, antecedentes personales de RDSM, epilepsia, TEA, dificultades conductuales, estereotipias, examen neurológico actual y estudios realizados previamente.

Para abordar el estudio se realiza una subdivisión de los pacientes en 3 grupos según sus características fenotípicas, lo que permitió una descripción dirigida y elección del estudio genético (figura 1).

\section{Grupo 1}

Pacientes con DI sin fenotipo específico. En este grupo de pacientes el examen clínico, las evaluaciones y los exámenes diagnósticos no evidencian indicadores etiológicos de la causa de DI y no presentan características fenotípicas especiales. Se estudian con CMA, examen que se utiliza como estudio de primera línea en $\mathrm{DI}^{13}$.

\section{Grupo 2}

Pacientes con DI asociado a fenotipo de desórdenes del neurodesarrollo con características clínicas de Síndrome de Angelman - Rett like. En este grupo se encuentran pacientes con características clínicas suge- rentes de un Síndrome de Angelman y/o Síndrome de Rett, destacando en su fenotipo clínico microcefalia, epilepsia y estereotipias. Los pacientes que no cumplen todos los criterios diagnósticos para clasificarlos como Síndrome de Angelman y/o Rett, o tienen estudios etiológicos que descartan estas patologías, se definen como Síndrome de Angelman - Rett like.

\section{Grupo 3}

Pacientes con DI y epilepsia de difícil manejo. En este grupo destaca en su fenotipo epilepsia que ha sido tratada con más de 3 fármacos antiepilépticos, descartándose causas estructurales con neuroimágenes y estudios previos que no han determinado etiología de DI.

Para los Grupos 2 y 3 se determinó utilizar paneles genéticos comerciales específicos diseñados para el estudio de pacientes con alteraciones del neurodesarrollo o epilepsia.

En el marco de este estudio se actualizaron evaluaciones de funcionamiento intelectual a pacientes con más de 1 año de la última evaluación, con instrumentos estandarizados para población chilena con escala de inteligencia de Wechsler. Se aplicó la escala de Wechsler versión V (WISC-V) para niños entre 6 y 16 años y Wechsler versión IV para adultos (WAIS-IV) a pacientes de 16 años en adelante. Se registró coeficiente intelectual total (CIT), sus índices con puntajes compuestos (PC) y calificaciones cualitativas (CC).

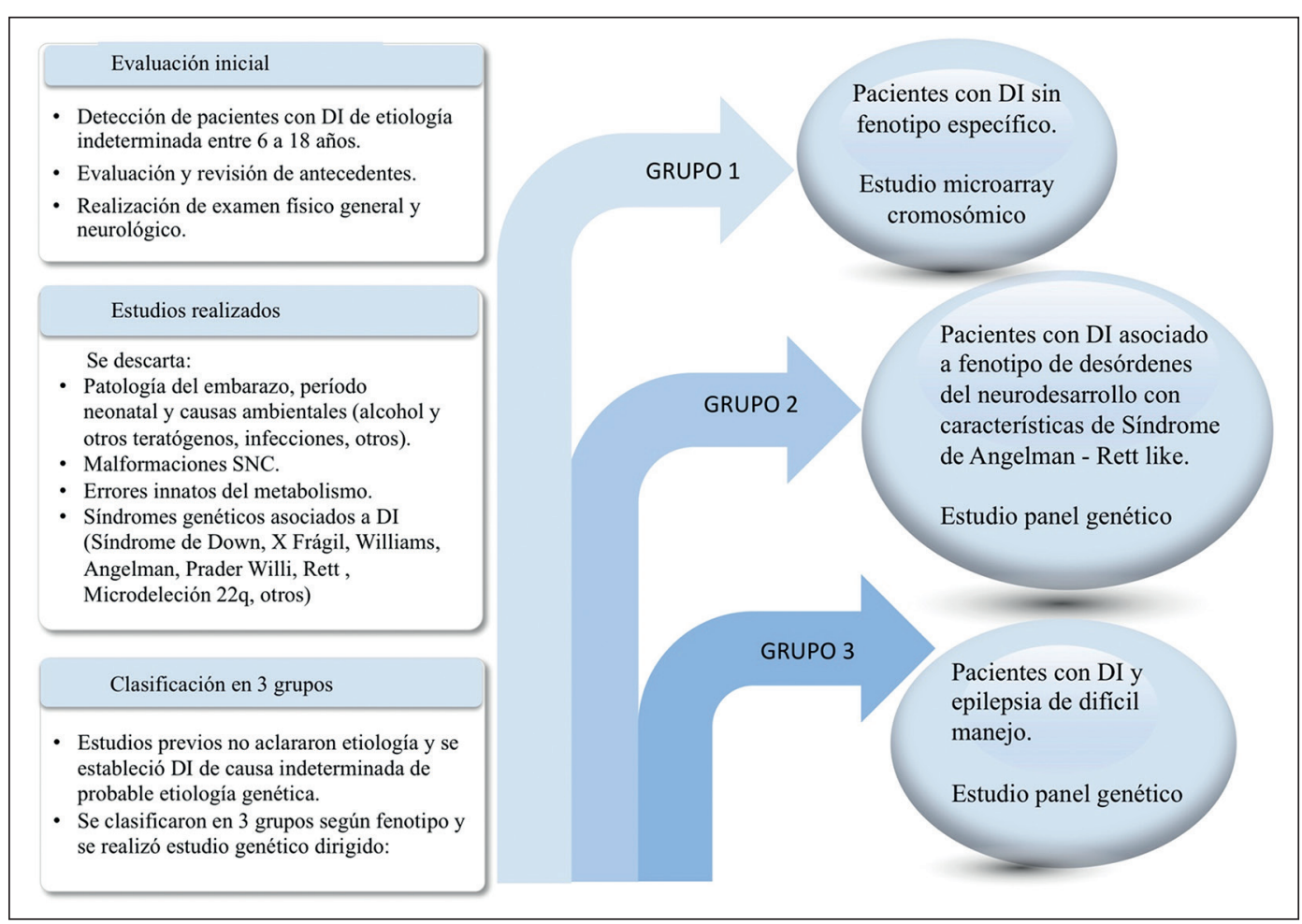

Figura 1. Algoritmo de derivación y estudio de pacientes con Discapacidad Intelectual (DI) de etiología indeterminada. 
En pacientes con severidad clínica que no pudieron ser evaluados de forma estandarizada con escala de Wechsler para obtener funcionamiento intelectual, se realizó evaluación con Test de Aprendizaje y Desarrollo infantil (TADI) permitiendo obtener edades de desarrollo ${ }^{17}$. Este instrumento permite evaluar desarrollo entre 3 meses y 6 años de edad, en dimensiones cognición, lenguaje, motricidad y socioemocional. Se informan puntajes brutos y tramos de edades correspondientes a las actividades que el paciente logra realizar en rangos etarios alcanzados en cada dimensión. Para el estudio se considera dimensión cognición y sus tramos de edades de desarrollo. Este test se aplicó como instrumento adaptado.

El estudio genético se programó de acuerdo al grupo en el que cada paciente fue clasificado. Los pacientes del Grupo 1 se estudiaron con análisis cromosómico de microarray (CMA) (centoarraycyto ${ }^{\mathrm{TM}_{-}} 750 \mathrm{~K}$ ) que permite detección de variaciones del número de copias (CNV) y/o deleciones/duplicaciones de gran tamaño, se reportan las CNV con un mínimo de 25 marcadores y un tamaño de más de $50 \mathrm{~kb}$ (deleciones) y $200 \mathrm{~kb}$ (duplicaciones). El Grupo 2 se estudió con panel genético de síndrome Rett/Angelman y desórdenes relacionados que incluye 28 genes, y el Grupo 3 se estudió con panel genético de epilepsia que incluye 187 genes. Los paneles genéticos se realizan con análisis de secuenciación y deleción/duplicación de los genes (tecnología Illumina). Los análisis fueron realizados en laboratorio de diagnóstico genético certificado.

Este estudio fue aprobado por el Comité Ético Científico del Servicio de Salud Metropolitano Central, con consentimiento informado de los padres de los participantes.

\section{Resultados}

Fueron evaluados 18 niños y adolescentes con DI de etiología previamente indeterminada. Los resultados se describen en cada grupo.

\section{Grupo 1: Pacientes con discapacidad intelectual sin fenotipo específico}

Se estudiaron 9 pacientes con edades entre 6 a 18 años con un promedio de 12 años, de predominio sexo masculino. En antecedentes clínicos, todos los pacientes tuvieron padres no consanguíneos y en 1 paciente se encontró antecedente de DI en la madre. El RDSM estuvo presente en los 9 pacientes, en la mayoría fue global. Presentaron comorbilidad neurológica con epilepsia no refractaria en 4 pacientes, 3 pacientes con rasgos de espectro autista y dificultades conductuales en 6 pacientes. En examen neurológico la mayoría fueron normocefálicos, sin compromiso piramidal, extrapira- midal o cerebeloso. Se aplicaron pruebas de Weschler a 4 pacientes y a 5 pacientes se aplica TADI.

En el análisis de los microarray cromosómico se encontró un resultado patogénico detectándose una microdeleción 16p11.2, en un paciente de 6 años de sexo masculino, con antecedente de RDSM global, que presentó DI severo, epilepsia no refractaria, rasgos del espectro autista, trastorno conductual y antecedentes de DI en la madre. El paciente fue evaluado con TADI determinándose una edad de desarrollo entre 30 a 36 meses (paciente no 5 ).

En un paciente se encontró una variación en el número de copias en región 11p12, de significado incierto y un paciente resulta portador de una deleción heterocigota en región 16q24.3 que se clasifica como CNV patogénica. Se obtuvieron resultados negativos en los otros 6 pacientes (tabla 1 ).

\section{Grupo 2: Pacientes con discapacidad intelectual asociado a fenotipo de desórdenes del neuro- desarrollo con características de Síndrome de Angelman - Rett like}

Se estudiaron 3 pacientes con edades entre 7 a 15 años con un promedio de 11 años, todas de sexo femenino, con microcefalia y estereotipias de línea media. En antecedentes clínicos destaca que fueron hijas de padres no consanguíneos y no presentaban antecedentes familiares de DI. El RDSM global estuvo presente en las 3 pacientes, una paciente tuvo epilepsia no refractaria y otra paciente presentó dificultades conductuales. Sólo una paciente tenía distonía. Se evalúan con TADI las 3 pacientes.

Se estudiaron con panel genético de Síndrome Rett/Angelman y desórdenes relacionados, obteniéndose una paciente con resultado de significado incierto y 2 pacientes con estudio negativo (tabla 2 ).

\section{Grupo 3: Pacientes con discapacidad intelectual y epilepsia de difícil manejo}

Se estudiaron 6 pacientes con DI y epilepsia de difícil manejo, con edades entre 6 a 14 años con un promedio de 10 años, de predominio sexo masculino. Ninguno tuvo antecedente de padres consanguíneos ni de DI en la familia. El RDSM está presente en los 6 pacientes, en la mayoría es global. Dos pacientes tuvieron dificultades conductuales y un paciente rasgos de TEA. En el examen clínico se encuentra microcefalia en un paciente, en otro paciente compromiso piramidal. Se evaluaron con TADI a 4 pacientes y con Weschler a 2 pacientes.

En el análisis genético con panel de epilepsia se obtuvo un resultado patogénico encontrándose una duplicación del gen IQSEC2, presente en un paciente masculino de 10 años con RDSM global, DI severo, TEA, dificultades conductuales, epilepsia y microcefa- 
Tabla 1. Descripición del Grupo 1: Características clínicas y resultado de estudio genético con microarray cromosómico en pacientes con discapacidad intelectual sin fenotipo específico.

\begin{tabular}{|c|c|c|c|c|c|c|c|c|c|}
\hline & & & & & $N^{\circ}$ Paciente & & & & \\
\hline & 1 & 2 & 3 & 4 & 5 & 6 & 7 & 8 & 9 \\
\hline Edad (años) & 16 & 10 & 10 & 8 & 6 & 8 & 18 & 17 & 18 \\
\hline Género & M & M & M & M & M & M & $\mathrm{F}$ & $\mathrm{F}$ & M \\
\hline Antecedente Familiar de DI & $(-)$ & $(-)$ & $(-)$ & $(-)$ & $(+)$ & $(-)$ & $(-)$ & $(-)$ & $(-)$ \\
\hline Consanguinidad & $(-)$ & $(-)$ & $(-)$ & $(-)$ & $(-)$ & $(-)$ & $(-)$ & $(-)$ & $(-)$ \\
\hline RDSM & $(+) \mathrm{G}$ & $(+) \mathrm{G}$ & $(+) G$ & $(+) \mathrm{PM}$ & $(+) G$ & $(+) G$ & $(+) G$ & $(+) G$ & $(+) \mathrm{PL}$ \\
\hline Epilepsia & $(-)$ & $(+)$ & $(-)$ & $(-)$ & $(+)$ & $(-)$ & $(+)$ & $(-)$ & $(+)$ \\
\hline Dificultades conductuales & $(+)$ & $(+)$ & $(+)$ & $(-)$ & $(+)$ & $(-)$ & $(+)$ & $(-)$ & $(+)$ \\
\hline TEA & $(+)$ & $(-)$ & $(+)$ & $(-)$ & $(+)$ & $(-)$ & $(-)$ & $(-)$ & $(-)$ \\
\hline Estereotipias línea media & $(-)$ & $(-)$ & $(-)$ & $(-)$ & $(-)$ & $(-)$ & $(+)$ & $(-)$ & $(-)$ \\
\hline Microcefalia/Macrocefalia & $\begin{array}{l}\text { Macro } \\
\text { cefalia }\end{array}$ & $(-)$ & $(-)$ & $(-)$ & $(-)$ & $(-)$ & $\begin{array}{l}\text { Micro } \\
\text { cefalia }\end{array}$ & $(-)$ & $\begin{array}{l}\text { Macro } \\
\text { cefalia }\end{array}$ \\
\hline Síndrome piramidal & $(-)$ & $(-)$ & $(-)$ & $(-)$ & $(-)$ & $(-)$ & $(-)$ & $(-)$ & $(-)$ \\
\hline Síndrome extrapiramidal & $(-)$ & $(-)$ & $(-)$ & $(-)$ & $(-)$ & $(-)$ & $(-)$ & $(-)$ & $(-)$ \\
\hline Síndrome cerebeloso & $(-)$ & $(-)$ & $(-)$ & $(-)$ & $(-)$ & $(-)$ & $(-)$ & $(-)$ & $(-)$ \\
\hline Estudio cognitivo Wechsler & $\begin{array}{c}\text { WISC-V } \\
\text { CIT } \\
\text { PC } 45 \text { / EB }\end{array}$ & - & - & - & - & $\begin{array}{c}\text { WISC-V } \\
\text { CIT } \\
\text { PC } 54 \text { / EB }\end{array}$ & - & $\begin{array}{c}\text { WISC-V } \\
\text { CIT } \\
\text { PC } 40 \text { / EB }\end{array}$ & $\begin{array}{l}\text { WAIS-IV } \\
\text { CIT } \\
55 / \mathrm{MB}\end{array}$ \\
\hline Estudio cognitivo TADI (meses) & - & 18 a 24 & 9 a 12 & 30 a 36 & 30 a 36 & - & 18 a 24 & - & - \\
\hline Estudio genético & Portador & $(-)$ & $(-)$ & $(-)$ & $(+)$ & $(-)$ & $(-)$ & Inc & $(-)$ \\
\hline Variante & $\begin{array}{l}\text { Deleción } \\
16 q 24.3\end{array}$ & $(-)$ & $(-)$ & $(-)$ & $\begin{array}{c}\text { Micro } \\
\text { deleción } \\
16 p 11.2\end{array}$ & $(-)$ & $(-)$ & $\begin{array}{l}\text { Duplicación } \\
\text { región } \\
11 \text { p12 }\end{array}$ & $(-)$ \\
\hline Clasificación de la variante & $\begin{array}{c}\text { CNV } \\
\text { patogénica }\end{array}$ & $(-)$ & $(-)$ & $(-)$ & $\begin{array}{c}\text { CNV } \\
\text { patogénica }\end{array}$ & $(-)$ & $(-)$ & VUS & $(-)$ \\
\hline
\end{tabular}

M: masculino; F: femenino; DI: discapacidad intelectual; (-) negativo; (+) positivo; RDSM: retardo del desarrollo psicomotor; G: global; PM: predominio motor; PL: predominio lenguaje; TEA: trastorno del espectro autista; TADI: valores de edad de desarrollo-dimensión cognición en meses; WISC-V: CIT: CI total; PC: puntaje compuesto; Calificación cualitativa: EB: extremadamente baja, MB: muy bajo. Inc: incierto; CNV: variante del número de copias; VUS: variante de significado incierto.

lia. El paciente fue evaluado con TADI, con dimensión cognición con rango etario entre 3 a 6 meses (paciente 13). En los otros 5 pacientes no se encontró etiología específica con el estudio realizado: 2 pacientes portadores en estado heterocigoto para variantes patogénicas y 3 pacientes con variantes de significado incierto (tabla 3).

\section{Discusión}

Se estudian niños y adolescentes con DI de etiología indeterminada en que se sospecha origen genético, encontrándose el antecedente de RDSM global en un alto porcentaje y la mayoría con condiciones neurológicas asociadas, con mayor frecuencia epilepsia y rasgos de TEA. En la serie la mayoría de los pacientes tuvo compromiso clínico severo y se les aplicó a gran parte del grupo la escala TADI adaptada como herramienta para determinar edades de desarrollo ${ }^{17,18}$.

Como resultado de los estudios realizados se encontró una causa de origen genético para DI en sólo 2 pacientes, un paciente con una microdeleción 16p11.2 de herencia $\mathrm{AD}$ detectado con microarray cromosómico y un paciente con una duplicación en el gen IQSEC2 detectado en el panel de epilepsia. En el fenotipo del Síndrome de microdeleción 16p11.2 se han descrito RDSM, DI y/o TEA, epilepsia, macrocefalia y obesi- 
Tabla 2. Descripción del Grupo 2: Características clínicas y resultado de estudio con panel genético de Síndrome Rett/Angelman y desórdenes relacionados en pacientes con discapacidad intelectual

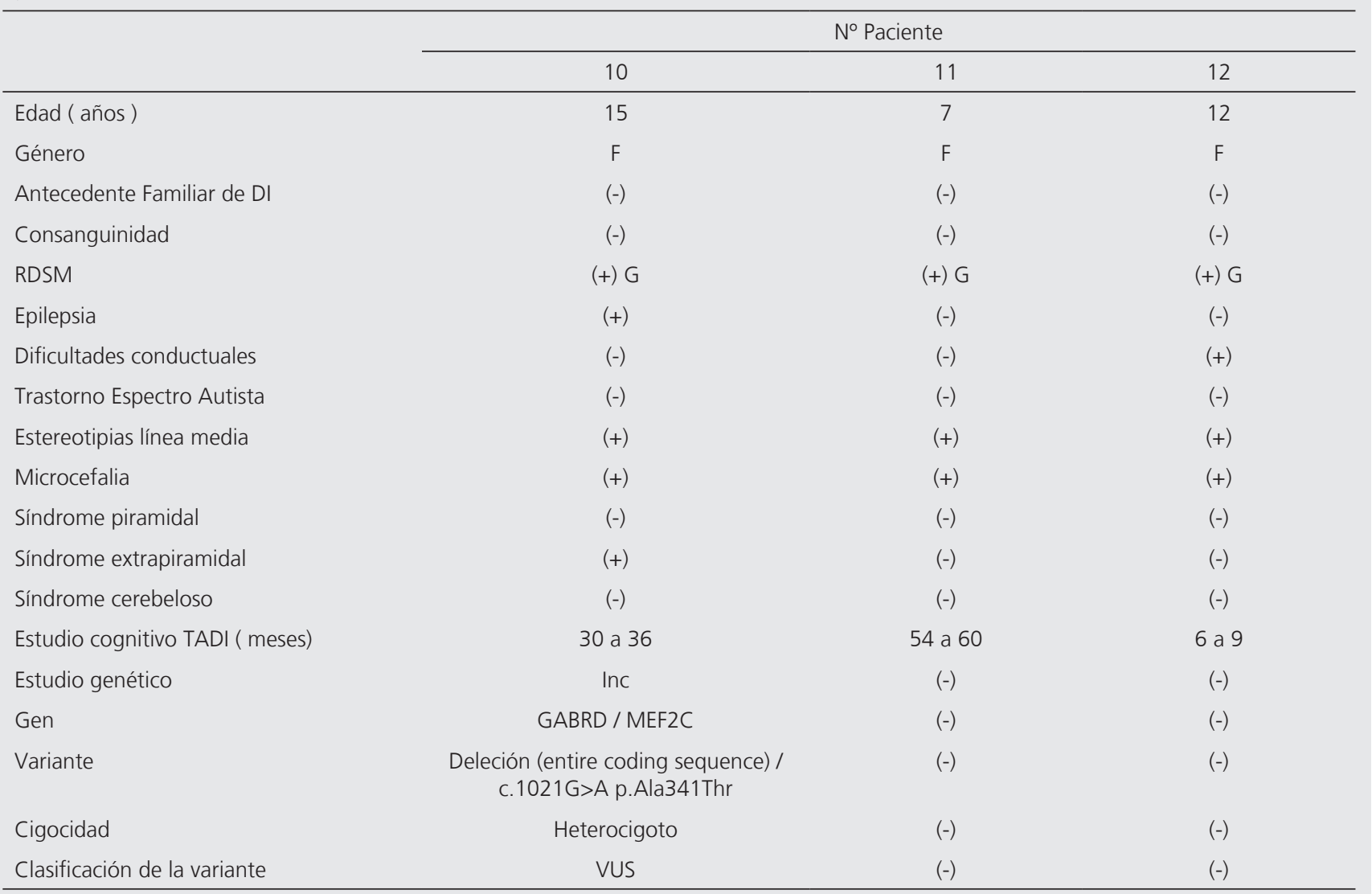

M: masculino; F: femenino; DI: Discapacidad intelectual; (-): negativo; (+): positivo; RDSM: retardo del desarrollo psicomotor; G: global; TADI: valores de edad de desarrollo-dimensión cognición en meses; Inc: incierto;VUS: variante de significado incierto

dad, es de herencia $\mathrm{AD}$, habitualmente de novo pero hasta en $20 \%$ puede ser heredado ${ }^{19,20,21}$. Los trastornos relacionados con IQSEC2 representan fenotipos con DI como característica principal con herencia ligada al cromosoma X. En el espectro de características clínicas se ha descrito en DI sindrómico y no sindrómico, se ha reportado en pacientes con DI severo y epilepsia, que pueden presentar estrabismo, hipotonía y rasgos autistas $^{22}$.

Se detectan en la serie pacientes portadores de variantes patogénicas heterocigotas y reportes de variantes de significado incierto, la mayor parte se encuentran en los pacientes del Grupo 3, con 2 pacientes portadores heterocigotos de variantes patogénicas y variantes de significado incierto en 3 pacientes. A esta información no es posible darle valor diagnóstico y se debe hacer seguimiento a las actualizaciones de las bases de datos en el tiempo y realizar estudio dirigidos a los padres, ya que estas variantes pueden ir reclasificándose $\mathrm{c}^{23}$.

El enfoque inicial del algoritmo diagnóstico y el clasificar a los pacientes en los 3 grupos descritos nos permitieron en nuestra experiencia orientar la elección del estudio genético según características clínicas de los pacientes con DI. En especial en la elección de los Grupos 2 y 3 se consideró que tanto los desórdenes del neurodesarrollo como Síndrome de AngelmanRett-like y la epilepsia de difícil manejo son fenotipos frecuentes en pacientes con DI, consideramos útil en primera instancia estudiar los genes más frecuentes encontrados en este tipo de condiciones los que se incluyen en los paneles correspondientes como una primera aproximación al diagnóstico etiológico desde el fenotipo clínico.

Futuros estudios son necesarios para ampliar el número de niños y adolescentes a evaluar, considerando además la realización de exámenes de nueva generación como análisis exómicos y/o genómicos que aumentan rendimiento diagnóstico. Como desventaja éstos últimos, así como los exámenes realizados en este estudio, tienen alto costo y no están implementados en el sistema público de salud, en consideración a esto se 
Tabla 3. Descripción del Grupo 3: Características clínicas y resultado de estudio con panel genético de epilepsia en pacientes con discapacidad intelectual

\begin{tabular}{|c|c|c|c|c|c|c|}
\hline & \multicolumn{6}{|c|}{ No Paciente } \\
\hline & 13 & 14 & 15 & 16 & 17 & 18 \\
\hline Edad ( años ) & 10 & 13 & 11 & 14 & 7 & 6 \\
\hline Género & M & M & M & $\mathrm{F}$ & M & $\mathrm{F}$ \\
\hline Antecedente Familiar de DI & $(-)$ & $(-)$ & $(-)$ & $(-)$ & $(-)$ & $(-)$ \\
\hline Consanguinidad & $(-)$ & $(-)$ & $(-)$ & $(-)$ & $(-)$ & $(-)$ \\
\hline RDSM & $(+) \mathrm{G}$ & $(+) G$ & (+) PM & $(+) \mathrm{PL}$ & $(+) \mathrm{G}$ & $(+) G$ \\
\hline Epilepsia & $\begin{array}{l}(+) \\
\mathrm{DM}\end{array}$ & $\begin{array}{l}(+) \\
\text { DM }\end{array}$ & $\begin{array}{l}(+) \\
\mathrm{DM}\end{array}$ & $\begin{array}{l}(+) \\
\mathrm{DM}\end{array}$ & $\begin{array}{l}(+) \\
\mathrm{DM}\end{array}$ & $\begin{array}{l}(+) \\
\mathrm{DM}\end{array}$ \\
\hline Dificultades conductuales & $(+)$ & $(-)$ & $(+)$ & $(-)$ & $(-)$ & $(-)$ \\
\hline Trastorno Espectro Autista & $(+)$ & $(-)$ & $(-)$ & $(-)$ & $(-)$ & $(-)$ \\
\hline Estereotipias línea media & $(-)$ & $(-)$ & $(-)$ & $(-)$ & $(-)$ & $(-)$ \\
\hline Microcefalia & $(+)$ & $(-)$ & $(-)$ & $(-)$ & $(-)$ & $(-)$ \\
\hline Síndrome piramidal & $(-)$ & $(+)$ & $(-)$ & $(-)$ & $(-)$ & $(-)$ \\
\hline Síndrome extrapiramidal & $(-)$ & $(-)$ & $(-)$ & $(-)$ & $(-)$ & $(-)$ \\
\hline Síndrome cerebeloso & $(-)$ & $(-)$ & $(-)$ & $(-)$ & $(-)$ & $(-)$ \\
\hline Estudio cognitivo Wechsler & - & - & - & $\begin{array}{c}\text { WISC-V } \\
\text { CIT } \\
\text { PC: } 59 / E B\end{array}$ & $\begin{array}{l}\text { WISC-III } \\
\text { DI leve }\end{array}$ & - \\
\hline Estudio cognitivo TADI (meses) & 3 a 6 & 9 a 12 & 6 a 9 & - & - & 30 a 36 \\
\hline Estudio genético & $(+)$ & Portador & Inc & Portador & Inc & Inc \\
\hline Gen & IQSEC2 & PIGG & SETD2 & TPP1 & GRLA1 & REL / REL \\
\hline Variante & $\begin{array}{c}\text { c.4402_4418dup } \\
\text { (p.Ser1474 } \\
\text { Alafs*27) }\end{array}$ & $\begin{array}{c}\text { Deleción } \\
\text { (entire coding } \\
\text { sequence) }\end{array}$ & $\begin{array}{l}\text { c.1664A>C } \\
\text { (p.Tyr555Ser) }\end{array}$ & $\begin{array}{c}\text { c. } 1424 C>T \\
\text { (p.Ser475Leu) }\end{array}$ & $\begin{array}{c}\text { c.31C>A } \\
\text { (p.Leu11\|le) }\end{array}$ & $\begin{array}{c}\text { c. } 2725 \mathrm{G}>\mathrm{A} \\
\text { (p.Ala909Thr)/ } \\
\text { c.7043G>A } \\
\text { (p.Gly2348Asp) }\end{array}$ \\
\hline Cigocidad & Homocigoto & Heterocigoto & Heterocigoto & Heterocigoto & Heterocigoto & Heterocigoto \\
\hline Clasificación de la variante & $\begin{array}{c}\text { CNV } \\
\text { patogénico }\end{array}$ & $\begin{array}{c}\text { CNV } \\
\text { patogénico }\end{array}$ & VUS & $\begin{array}{c}\text { CNV } \\
\text { patogénico }\end{array}$ & VUS & VUS \\
\hline
\end{tabular}

M: masculino; F: femenino; DI: Discapacidad Intelectual; (-): negativo; (+): positivo; RDSM: retardo del desarrollo psicomotor; G: global; PM: predominio motor; PL: predominio lenguaje; DM: difícil manejo; TEA: trastorno del espectro autista; WISC-V: CIT: CI total; PC: puntaje compuesto; CC: calificación cualitativa; EB: extremadamente baja; TADI: valores de edad de desarrollo-dimensión cognición en meses; Inc: incierto; CNV: variante del número de copias; VUS: variante de significado incierto

hace aún mas necesario optimizar los estudios genéticos disponibles.

Encontrar la etiología en DI permite al clínico detectar comorbilidades, orientar al manejo y tratamientos específicos del paciente y otorgar un consejo genético. Las familias que han conocido la causa de DI destacan que el diagnóstico permitió conocer mejor la condición de su hijo y obtener apoyos particulares. Es un desafío además de conocer la causa el profundizar en el conocimiento de los ámbitos de conducta adapativa y calidad de vida en pacientes con DI.

\section{Conclusión}

Esta serie clínica describe aspectos clínicos, evaluaciones cognitivas y resultados de estudios genéticos específicos elegidos de acuerdo al fenotipo clínico de niños y adolescentes con DI de etiología previamente indeterminada, donde se logra precisar la causa genética de DI en 2 pacientes. Resulta de utilidad el abordaje del estudio genético según la presencia de características fenotípicas asociadas a DI que permite guiar el estudio y racionalizar los recursos que actualmente 
son limitados en nuestro sistema público de salud. Conocer la causa de DI permite a la familia un mejor conocimiento de la condición del paciente y al equipo de salud realizar en forma dirigida el manejo, seguimiento $\mathrm{y}$ adecuaciones pertinentes en forma individualizada.

\section{Responsabilidades Éticas}

Protección de personas y animales: Los autores declaran que los procedimientos seguidos se conformaron a las normas éticas del comité de experimentación humana responsable y de acuerdo con la Asociación Médica Mundial y la Declaración de Helsinki.

Confidencialidad de los datos: Los autores declaran que han seguido los protocolos de su centro de trabajo sobre la publicación de datos de pacientes.

Derecho a la privacidad y consentimiento informado: Los autores han obtenido el consentimiento informado de los pacientes y/o sujetos referidos en el artículo. Este documento obra en poder del autor de correspondencia.

\section{Conflicto de intereses}

Los autores declaran no tener conflicto de intereses.

\section{Financiamiento}

Esta investigación fue financiada como proyecto de estudio, por fondos aportados por el Grupo de Trabajo en Discapacidad Intelectual y Salud de la Facultad de Medicina de la Universidad de Chile.

\section{Agradecimientos}

Agradecemos a nuestros pacientes y sus familias, así como al equipo multidisciplinario de Salud del Servicio de Neuropsiquiatría Infantil del Hospital San Borja Arriarán.

\section{Referencias}

1. American Psychiatric Asociation, Manual Diagnóstico y Estadístico de los Trastornos Mentales $5^{\mathrm{a}}$ edición, DSM 5, Arlington, VA Editorial Médica Panamericana S.A. Sección Trastornos del desarrollo neurológico. Discapacidades intelectuales 17;17-22.

2. Schalock R, Borthwick D, Sharon A, et al. Intellectual Disability: Definition, Classification, and Systems of Supports. Eleventh Edition 2010. https://eric. ed.gov/?id=ED509596.

3. Amador JA, Forns M. Escala de inteligencia de Wechsler para niños, WISC-V, quinta edición. Facultad de Psicología. Universidad de Barcelona 2019.

4. Rosas R, Tenorio M, Pizarro M, et al. Estandarización de la Escala Wechsler de Inteligencia Para Adultos Cuarta Edición en Chile. Psykhe [online]. 2014;vol.23(1): 1-18 [citado 2021-01-24]. Disponible en: <https://scielo.conicyt.cl/scielo.php? script $=$ sci_arttext\&pid=S07182228201 $4000100001 \& \operatorname{lng}=\mathrm{es} \& \mathrm{nrm}=\mathrm{iso}>$.ISSN 0718-2228. http://dx.doi.org/10.7764/ psykhe.23.1.529.

5. Rodríguez M, Rosas R, Pizarro M. Rendimiento en escala WISC-V en población urbana y rural de Chile. 2019. (CEDETI UC Papeles de Investigación $\left.\mathrm{N}^{\circ} 11\right)$.

6. Brown K, Parikh S, Patel D. Understanding basic concepts of developmental diagnosis in children, Transl Pediatr. 2020;9(Suppl 1):S9-S22.

7. Vasudevan P and Suri M. A clinical approach to developmental delay and intellectual disability, Clinical Medicine 2017; Vol 17( N 6):558-61.

8. Purugganan O. Intellectual Disabilities, Pediatrics in Review 2018;39:299. DOI: 10.1542/pir.2016-0116

9. Lee K, Cascella M, Marwaha R. Intellectual Disability. [Updated 2020 Nov 29]. In: StatPearls [Internet]. Treasure Island (FL): StatPearls Publishing 2020 Jan-. Available from: https://www.ncbi. nlm.nih.gov/books/NBK547654/

10. Chiurazzi P, Pirozzi F. Advances in understanding - genetic basis of intellectual disability. F1000Res. 2016 Apr 7;5:F1000 Faculty Rev-599. doi: 10.12688/ f1000research.7134.1. PMID: 27127621; PMCID: PMC4830215

11. Vissers LE, Gilissen C, Veltman JA Genetic studies in intellectual disability and related disorders. Nat Rev Genet. 2016 Jan;17(1):9-18. doi: 10.1038/ nrg3999. Epub 2015 Oct 27. PMID: 26503795.

12. Flore LA and Milunsky JM. Genetic evaluation of child with global developmental delay or intellectual disability; Semin Pediatr Neurol. 2012;19:173-80.

13. Miller D, Adam M, Aradhya S, et al; Consensus Statement: Chromosomal Microarray Is a First-Tier Clinical Diagnostic Test for Individuals with Developmental Disabilities or Congenital Anomalies. Am J Hum Genet. 2010;86(5):749-64. doi: 10.1016/j. ajhg.2010.04.006.

14. Lee JS, Hwang H, Kim SY, et al. Chromosomal Microarray With Clinical Diagnostic Utility in Children With Developmental Delay or Intellectual Disability. Ann Lab Med. 2018 Sep;38(5):473-80. doi: 10.3343/ alm.2018.38.5.473.

15. Jang W, Kim Y, Han E, et al. Chromosomal Microarray Analysis as a First-Tier Clinical Diagnostic Test in Patients With Developmental Delay/ Intellectual Disability, Autism Spectrum Disorders, and Multiple Congenital Anomalies: A Prospective Multicenter Study in Korea. Ann Lab Med. 2019;39:299-310.

16. Vickers R, Gibson JS. A Review of the Genomic Analysis of Children Presenting with Developmental Delay/Intellectual Disability and Associated Dysmorphic Features. January 12, 2019;11(1):e3873. DOI 10.7759/cureus.3873.

17. Edwards M, Pardo M, Gómez M. Test de aprendizaje y desarrollo infantil (TADI) para niñas y niños de 3 meses a 6 años; primera edición. UNICEF, Santiago 2012.

18. Tenorio M, Bunster J, Arango P, et al. Experiencia de confiabilidad y validez del Test de Aprendizaje y Desarrollo Infantil (TADI) para evaluación niñas y niños chilenos con Síndrome de Down, Psykhe 2020;29(1):1-16. 
19. Han JY, Lee IG. Genetic tests by nextgeneration sequencing in children with developmental delay and/or intellectual disability. Clin Exp Pediatr. 2020;63(6):195-202. doi: 10.3345/ kjp.2019.00808.

20. Goldenberg P. An Update on Common Chromosome Microdeletion and
Microduplication Syndromes, Pediatric Annals, 2018 1;47(5):e198-e203.

21. Miller DT, Chung W, Nasir R, et al. 16p11.2 Recurrent Microdeletion. GeneReviews ${ }^{\circledR}$ [updated 2015 Dec 10]. https://www.ncbi.nlm.nih.gov/books/ NBK11167/.

22. Alexander-Bloch A, McDougle C,
Ullman Z, et al. IQSEC2 and X-linked intellectual disability, Psychiatric Genetics 2016;26:101-8.

23. Duzkale H, Shen J, McLaughlin H, et al. A Systematic approach to assessing the clinical significance of genetic variants. Clin Genet. 2013;84(5):453-63. doi: $10.1111 /$ cge. 12257 . 